\title{
EVALUATION OF THE TERMINAL SEQUENCING AND SPACING SYSTEM FOR PERFORMANCE-BASED NAVIGATION ARRIVALS
}

\author{
Jane Thipphavong, Dr. Jaewoo Jung, Harry Swenson, NASA Ames Research Center, Moffett Field, CA \\ Dr. Lynne Martin, San Jose State University, San Jose, CA \\ Melody Lin, Jimmy Nguyen, Optimal Synthesis, Los Altos, CA
}

\begin{abstract}
NASA has developed the Terminal Sequencing and Spacing (TSS) system, a suite of advanced arrival management technologies combining timebased scheduling and controller precision spacing tools. TSS is a ground-based controller automation tool that facilitates sequencing and merging arrivals that have both current standard ATC routes and terminal Performance-Based Navigation (PBN) routes, especially during highly congested demand periods. In collaboration with the FAA and MITRE's Center for Advanced Aviation System Development (CAASD), TSS system performance was evaluated in human-in-the-loop (HITL) simulations with currently active controllers as participants. Traffic scenarios had mixed Area Navigation (RNAV) and Required Navigation Performance (RNP) equipage, where the more advanced RNP-equipped aircraft had preferential treatment with a shorter approach option. Simulation results indicate the TSS system achieved benefits by enabling $\mathrm{PBN}$, while maintaining high throughput rates-10\% above baseline demand levels. Flight path predictability improved, where path deviation was reduced by $2 \mathrm{NM}$ on average and variance in the downwind leg length was $75 \%$ less. Arrivals flew more fuel-efficient descents for longer, spending an average of 39 seconds less in step-down level altitude segments. Self-reported controller workload was reduced, with statistically significant differences at the $p<0.01$ level. The RNP-equipped arrivals were also able to more frequently capitalize on the benefits of being "Best-Equipped, BestServed" (BEBS), where less vectoring was needed and nearly all RNP approaches were conducted without interruption.
\end{abstract}

\section{Introduction}

The United States Next Generation Air Transportation System (or NextGen) is being designed to support the predicted increases in traffic volume and to increase the capacity, efficiency and safety of the National Airspace System (NAS). The Federal Aviation Administration (FAA) identifies Performance-Based Navigation (PBN) as a key enabling capability in NextGen and is actively developing and implementing $\mathrm{PBN}$ procedures and routes at major airports nationwide [1].

PBN defines aircraft performance requirements in terms of navigation specifications. There are two kinds of navigation specifications: Area Navigation (RNAV) and Required Navigation Performance (RNP). RNP is a higher-fidelity RNAV specification with the addition of on-board performance monitoring and alerting as part of the avionics functionality. Aircraft equipped with RNP can fly procedures that are contained within a tightly defined corridor of airspace, which would increase predictability of flight paths to assist in the planning of efficient flows. To incentivize RNP aircraft equipage, these aircraft may have preferential routing with shortened flight distance or reduced separation requirements. Over $90 \%$ of commercial jets are already RNAV-equipped and less than half have advanced RNP equipage [2].

PBN Standard Terminal Arrival Routes (STAR), procedures, and approaches are designed to be fuelefficient optimal profile descents (OPDs) and also account for unique operational requirements such as avoiding terrain or other obstacles, de-conflicting airspace, or resolving environmental constraints [3]. PBN arrivals have been shown to increase efficiency and reduce delays, but their use is limited during periods of high traffic demand due to the complexity of merging multiple streams of aircraft to the same airport. Arrivals in the Terminal Radar Approach Control (TRACON) area are still primarily controlled using radar vectoring and step-down descents, resulting in high workload for controllers and preventing efficient $\mathrm{PBN}$ aircraft trajectories from being followed.

The FAA has recognized the need for a merging and spacing decision support system in the terminal 
area to achieve the full benefits of PBN, especially during periods of high traffic demand [3]. MITRE and NASA have prototyped terminal controller decision support tools to support PBN by enhancing functionalities in automation systems currently used in the field to facilitate near-term implementation.

MITRE initially developed the Spacing of Performance-based Arrivals on Converging Routes (SPACR) concept that expanded the capabilities of the Converging Runway Display Aid (CRDA) embedded in the current terminal automation system, Standard Terminal Automation Replacement System (STARS) [4]. CRDA was originally designed to facilitate proper spacing for aircraft conducting Dependent Converging Instrument Approaches (DCIAs) by projecting a 'ghost' target on the trailing aircraft's path based on the leading aircraft's current position [5]. The CRDA capabilities were extended to also coordinate upstream merges in the terminal area and developed into a passive situation awareness aid called the Relative Position Indicator (RPI) [6-7] RPI has had favorable feedback from controllers in assisting merging of RNAV flows as well as potential delay reduction [8-9]. The RPI tool is purely geometry-based and does not account for the effects of winds and the speed deceleration of the ghost target aircraft flight path [10]. These effects result in ghost target position errors and, depending on the magnitude of the errors, may increase the number of controller interventions and oscillating speed adjustments [11].

NASA has researched a 4-D trajectory-based approach to managing PBN arrivals and developed the Terminal Sequencing and Spacing (TSS) system [12]. The TSS system is based on 4-D aircraft trajectory predictions that incorporate both wind estimates and speed profiles along flight paths. The two main components are: 1) a scheduler that deconflicts merging arrivals in the terminal area by computing appropriate arrival times to the runway threshold and upstream merge points, and 2) a set of Controller-Managed Spacing (CMS) dec ision support tools to efficiently assist schedule adherence [13]. The TSS scheduling component was created by extending the capabilities of the original Traffic Management Advisor (TMA) system currently used at Air Route Traffic Control Centers (or 'Centers') for terminal metering (TM) operations. This system, referred to as TMA-TM, has a synchronized schedule controlled by Center and TRACON, allowing for an integrated arrival management solution from en route cruise to the runway threshold. The TSS system has been tested in a number of human-in-the-loop (HITL) simulations, where results indicated high controller use and acceptability of the CMS tools as well as improved RNAV route conformance [14-15]. The robustness of the TSS system has also been investigated, specifically off-nominal recovery methods and mixed RNAV/RNP equipage situations at a mid-sized airport with rigid airspace constraints [16-18].

In the Spring of 2013, NASA, FAA and MITRE's CAASD conducted a joint HITL simulation to assess the effectiveness of the TSS system in enabling PBN operations performed by currently active Certified Professional Controllers (CPCs). The major contribution of this paper is quantifying TSS system performance using generalized heavy arrival demand scenarios to a major airport, with mixed RNAV/RNP equipage under realistic wind forecasts. The performance and workload of controller participants who had little to no experience using TSS prior to the simulations were compared to controller participants who had more experience with TSS, which provided insight into the effects of additional experience.

The paper is organized as follows: The next section further details the TSS system components and its operational concept. The following section describes the experimental details of the HITL simulations conducted at NASA Ames. Results from the simulations are discussed in the subsequent section, which evaluates system performance metrics, learning effects, and controller feedback of the TSS system. The last section concludes with a summary of key findings and plans for further research and development.

\section{Terminal Sequencing and Spacing System}

The TSS operational concept focuses on arrivals prior to top of descent (TOD) in Center airspace about $150 \mathrm{NM}$ from the TRACON boundary. The majority of aircraft are assumed RNAV-equipped, and some have advanced RNP capabilities. Aircraft navigate along published PBN routes that include runway transitions that may connect to the threshold. The TMA-TM generates an arrival schedule that 
conditions the flow in the Center to facilitate sequencing and spacing in the TRACON. The arrival schedule is broadcast to the Center and TRACON automation systems. To assist Center controllers with metering operations, their radar displays show meter lists and delay countdown timers (DCTs) with a resolution of tenths of minutes. TRACON controllers are presented with CMS advisory tools to assist schedule conformance. The following subsections describe the TMA-TM and CMS tools in further detail.

\section{Traffic Management Advisor with Terminal Metering Capabilities}

The TMA-TM is a strategic and tactical groundbased arrival management system that generates an arrival schedule as well as TRACON advisories for schedule conformance [12]. TMA-TM uses 4-D trajectory predictions to compute the arrival sequence, Scheduled Times-of-Arrival (STA) and runway assignments. Runway assignments are selected to balance runway usage and minimize overall system delay. The arrival sequence and STAs are computed at meter fixes located near the TRACON boundary, metering points in the terminal area where arrival flows merge, and the runway threshold. The STAs are designed to de-conflict arrivals at merge points and adhere to separation requirements. Delays to meet the STAs are allocated along the arrival route such that arrivals are able to remain on their assigned $\mathrm{PBN}$ routes in the terminal area. These schedule settings are dependent on the airspace geometry.

\section{Controller-Managed Spacing Tools}

The TMA-TM produces TRACON advisories based on the generated arrival schedule [19]. The CMS toolset provides the controller display aids for sequencing and spacing in the TRACON. Figure 1 shows the different types of CMS tools that can be displayed on the TRACON controller's radar display. The CMS tools provide a slot marker circle and the marker's Indicated Airspeed (IAS), the aircraft's IAS, TMA-TM runway assignment, sequence number, speed advisories, early/late $(\mathrm{E} / \mathrm{L})$ indicators, and timelines to assist metering operations in the terminal area. The circular slot marker provides a spatial reference for each aircraft, which considers the forec ast wind field, published restrictions, and the STA. The slot marker traverses the aircraft's STA trajectory. To follow the slot marker, a speed advisory is given to the next meter point along the

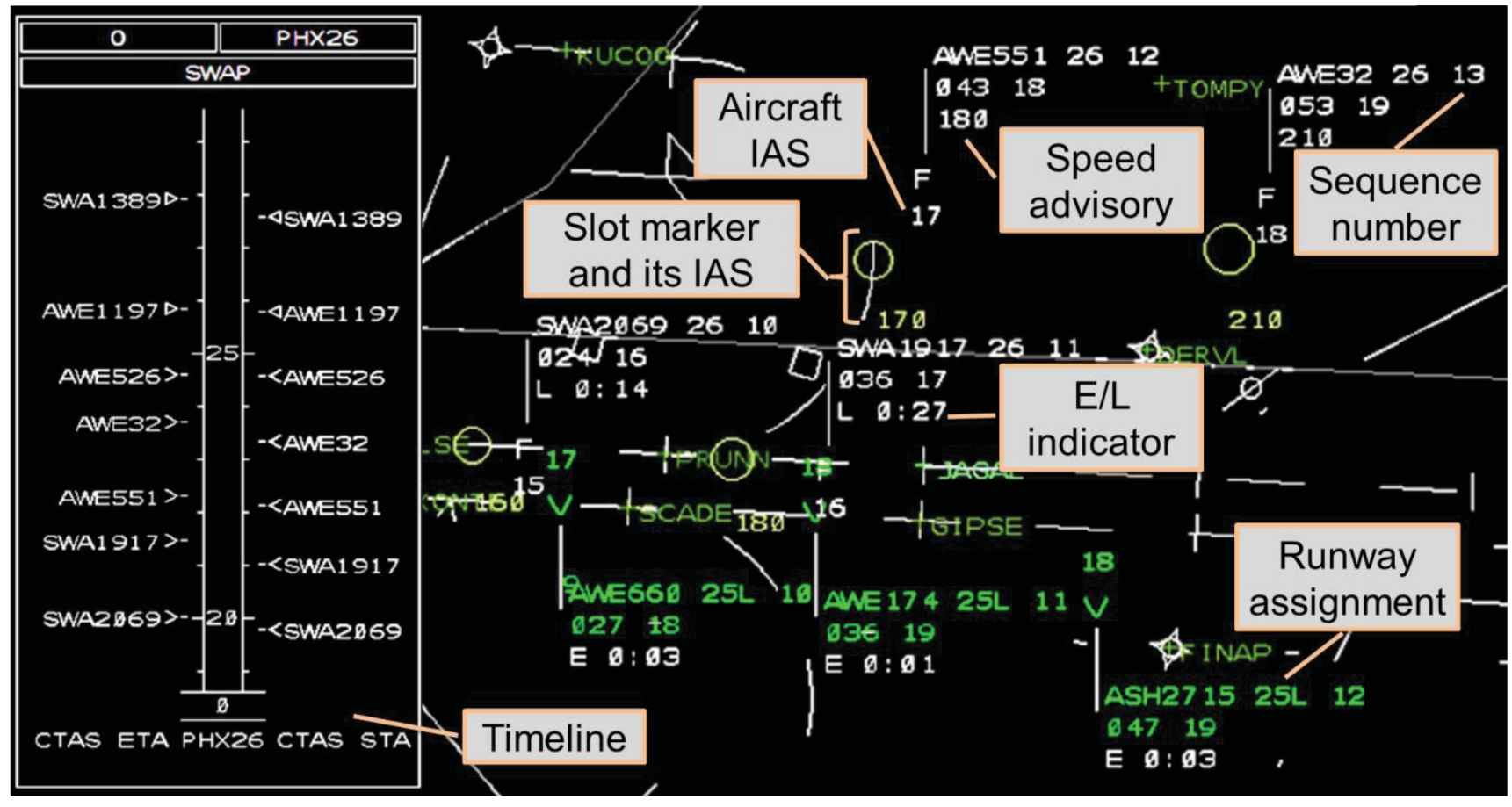

Figure 1. Controller-Managed Spacing toolset. 
arrival route. In cases where speed advisories are not sufficient for the aircraft to absorb the delay needed to meet its STA, an E/L indicator is displayed. Timelines are also available for the controller to quickly monitor arrival sequence, current demand loads, and delay values.

\section{Experiment Design}

The TSS system was tested at the NASA Ames Research Center air traffic control (ATC) simulation facility over a period of three weeks. The planning, coordination and execution of the simulation was a collaborative effort between NASA, FAA, and MITRE. The main objective of the simulation was to evaluate the TSS system that enabled PBN operations in scenario conditions with mixed RNAV/RNP equipage, realistic wind forecast errors, and heavy traffic. A secondary objective was to gain insight on the learning effects of additional experience and training of the TSS system.

\section{Simulation Environment}

The Multi-Aircraft Control System (MACS) was used to conduct the HITL simulations [20]. MACS provides high-fidelity operational radar display emulations for air traffic controllers as well as user interfaces and displays for confederate pilots and experiment managers. MACS also has a dynamic real-time air traffic simulation capability designed to generate realistic aircraft trajectories and associated radar messages for aircraft in a simulated airspace environment. The TMA-TM received aircraft flight plan information from MACS as input. The arrival schedule and TRACON advisories were then sent to MACS for the controller displays. The CMS controller display aids were integrated with the MACS emulation of the TRACON STARS display.

\section{Airspace}

The simulation airspace was Phoenix Sky Harbor International Airport (PHX) in the West Flow configuration, using runways $25 \mathrm{~L}$ and 26 . Simulated aircraft flew Instrument Flight Rules (IFR) and independent runway operations were as sumed. Figure 2 illustrates the RNAV/RNP OPD STARs and controller sector boundaries modeled in the simulation. Crossing restrictions were similar to those published in the FAA Terminal Procedures Publication.

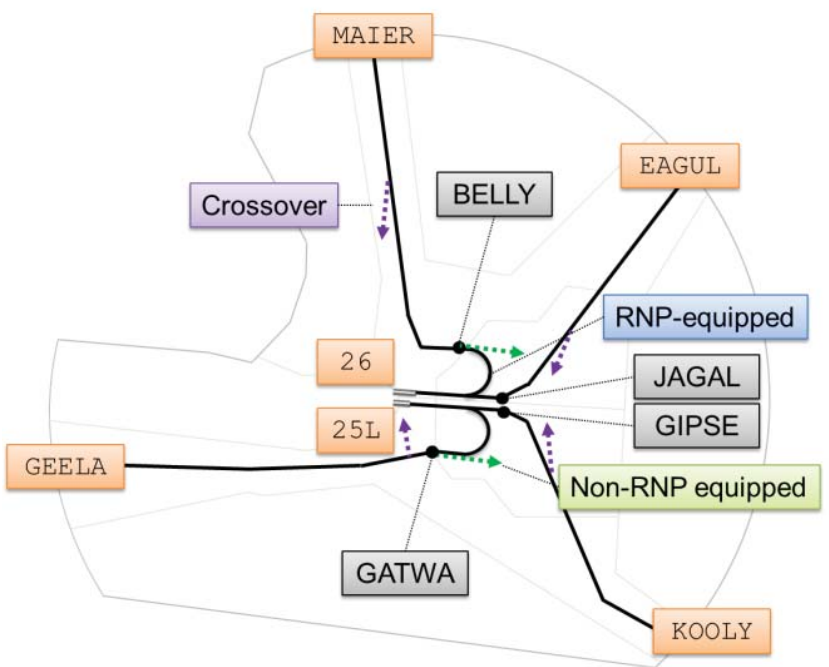

Figure 2. PHX airspace.

Albuquerque Center (ZAB) and Phoenix TRACON (P50) are primarily responsible for sequencing arrivals into PHX. The simulation had four ZAB arrival sectors, one for each meter fix, and four P50 positions, where a Feeder and Final pair controlled the North and South flows to each of the runways.

Arrival routes were assigned based on equipage level. RNAV-equipped arrivals using the MAIER and GEELA STARs have published routes ending at the BELLY and GATWA waypoints. These arrivals remained on the downwind leg until ATC clearance for the base leg turn, joining final near JAGAL or GIPSE. Some aircraft that have advanced RNP equipage are capable of precise fixed radius paths within $0.1 \mathrm{NM}$ lateral accuracy. With this capability, RNP-equipped arrivals were given the advantage of a shortened downwind route. Instead of joining the final by JAGAL or GIPSE, they could be cleared for the RNP-Authorization Required (RNP-AR) instrument approach procedure that intercepts the final 3-4 NM earlier. The RNP-AR approach must be cleared 16 NM West of BELLY or GATWA and is defined by the radius-to-fix (RF) leg intercepting final. Aircraft using the RNP-AR approach saved approximately $9 \mathrm{NM}$ of flight distance (i.e, $\sim 3$ minutes of flying time) compared to the conventional final intercept. Non-RNAV routes (not shown in Fig. 
2) were also defined, and assigned to lowerperformance jets and turboprops.

Arrivals are typically cleared for the runway located on the same side of the airspace (i.e., EAGUL and MAIER on 26, KOOLY and GEELA on 25L). However, the TMA-TM runway balancing algorithm may determine that landing on the other runway may be more efficient for the overall system. In cases where crossovers to the other runway occurred, arrivals on the MAIER and GEELA STARs were vectored over the airport (ref. Fig. 2) to join the downwind for the other runway or had an extended base leg crossover from the EAGUL or KOOLY STARs.

\section{Scenarios}

Three scenarios were used during data collection. Only arrivals were modeled for this simulation. Departure and satellite traffic will be added in future HITL simulations to increase fidelity. Scenario A was based on the current PHX morning arrival rush traffic pattern and aircraft mix, with $10 \%$ increased traffic demand. Scenarios B-1 and B-2 were designed to represent generalized scenarios with sustained, heavy traffic, having demand peaks similar to other busy national airports. Figure 3 plots the initial demand at the runways of each scenario for comparison. Table 1 summarizes the equipage variation and runway demand.

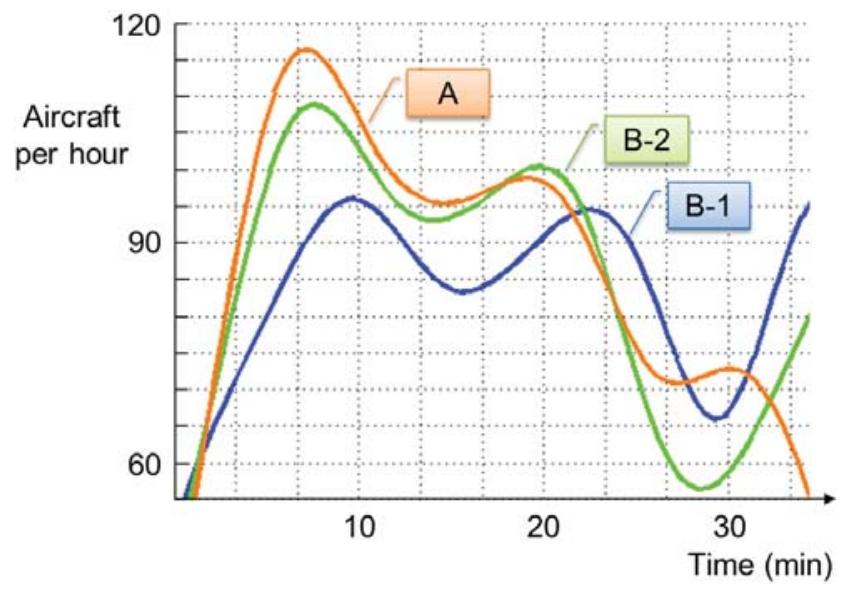

Figure 3. Initial demand rate at the runways.
Table 1. Number of aircraft in each scenario (N), percentage RNAV/RNP-equipped and default runway $25 \mathrm{~L}$.

\begin{tabular}{|c|c|c|c|c|}
\hline Scenario & N & \% RNAV & \% RNP & 25L \\
\hline A & 54 & $70 \%$ & $19 \%$ & $56 \%$ \\
\hline B-1 & 63 & $67 \%$ & $29 \%$ & $49 \%$ \\
\hline B-2 & 65 & $62 \%$ & $34 \%$ & $48 \%$ \\
\hline
\end{tabular}

\section{Controller Participants}

Four Center and four TRACON positions were staffed during the simulations. The Center controller participants were recently retired from Los Angeles and Albuquerque Center, each with more than 25 years of ATC experience.

There were two groups of TRACON controller participants with varying levels of experience using the TSS system. One group of participants had considerable knowledge of the TSS system, with four to 16 weeks of exposure from prior HITL simulations held at NASA. They were recently retired terminal controllers from P50 and Southern California (SoCal) TRACON, each with more than 25 years of experience.

The second group of TRACON participants had less experience using the TSS system. The group consisted of one recently retired controller and three currently active Certified Professional Controllers (CPCs) from Boston, Phoenix and New York TRACONs. The recently retired controller had 20 years of ATC experience, of which 10 were in the SoCal TRACON. The remaining three participants had at least 20 years of TRACON experience. The Phoenix and Boston CPCs had no exposure to the TSS system prior to the simulations. The others had less than two weeks of experience. Prior to data collection, they had 1.5 days of classroom and laboratory training on the airspace and the TSS system. During training, the first group of participants, with more TSS experience, coached the second group on the use of the advisory tools and addressed any simulation inquiries. 


\section{Test Conditions}

The test conditions varied the scenario, wind forecast and set of controller participants. The wind forecast used in the TMA-TM trajectory calculations either matched the truth winds or were 'mismatched,' having a root-mean-square difference of approximately 8.5 knots, and were consistent with measured wind forecast errors [21]. Scheduled delay in the TRACON varied between 20-35 seconds and an average of 2 minutes in the Center. Sc enarios B-1 and B-2 were run with the currently active CPCs only. In these runs, the Feeder and Final Pair rotated positions. Scenario A was used for both groups of controller participants to investigate the effects of differing controller techniques and expertise levels using the TSS toolset. All test conditions had baseline runs, where the TSS system was not used. Table 2 shows the number of runs for each test condition configuration. The TSS experience level of the participants are also listed, where one set was more 'Experienced' and the other set was 'New' to the system.

Table 2. Number of runs for each test condition.

\begin{tabular}{|c|c|c|c}
\hline Scenario & Participants & Wind Forecast & Runs \\
\hline \multirow{3}{*}{ A } & New & Mismatched & 2 \\
\cline { 3 - 4 } & \multirow{2}{*}{ Experienced } & Matched & 3 \\
\cline { 3 - 4 } & & Mismatched & 3 \\
\hline \multirow{2}{*}{ B-1 } & \multirow{2}{*}{ New } & Matched & 3 \\
\cline { 3 - 4 } & & Mismatched & 3 \\
\hline \multirow{2}{*}{ B-2 } & \multirow{2}{*}{ New } & Matched & 3 \\
\cline { 3 - 4 } & & Mismatched & 3 \\
\hline
\end{tabular}

\section{Controller and Pilot Procedures}

Center controllers managed arrivals starting approximately $70 \mathrm{NM}$ before TOD. They were responsible for meeting STAs at the meter fix and issuing the 'descend via' clearance for the RNAV OPD as defined in the FAA JO 7110.65U. In conditions where the TSS system was used, they also assigned the expected runway transition as determined by the TMA-TM scheduler. All controller and pilot interactions were via voice communication. Pseudo-pilots entered ATC advisories into the flight simulation user interface.
In the terminal area, Feeder Controllers were encouraged to keep arrivals on the RNAV OPD routes as long as possible and use the TSS advisory tools when available. They also coordinated, initiated and issued the heading advisories for arrivals using the over-the-airport crossover routes. Final Controllers were responsible for merging arrivals to final approach. RNAV-equipped aircraft on the downwind leg were advised when to turn onto final by ATC. RNP-equipped arrivals were cleared for the RNP-AR approach if feasible. RNP-equipped aircraft that used the over-the-airport crossover route were not eligible for the RNP-AR approach. Aircraft conducting the RNP-AR approach could be taken off course at any time for controller perceived safety or separation issues.

\section{Data Collection}

Data collection occurred over a period of three weeks. The first group of participants, who had advanced knowledge of the TSS system, completed data collection runs during the first week. The second group of participants, who had limited exposure to the TSS system, completed data collection runs over the second two weeks. Aircraft information, TRACON advisories and track data were recorded. Video footage of the controller displays was captured. All pilot/controller entries and voice communications were logged. Questionnaires were administered after each scenario run and at the end of each week.

\section{Results}

Results first present an assessment on the effectiveness of the TSS system in enabling PBN operations. Data in this section are from simulation runs using Scenarios B-1 and B-2 and currently active CPCs as participants. The next section focuses on characterizing the effects of additional training and familiarization with the TSS system. In this case, data are from two sets of controller participants using Scenario A. Controller workload metrics and feedback are examined in the last section. Overall results indicated that the change in wind forecasts did not have an influence on the data. Controller feedback also noted that it was easy to factor in the winds and that there was no anomalous impact on their control strategy. Thus, data from test conditions that differed only by wind conditions were combined. 


\section{Enabling PBN Operations}

The lateral paths of the RNAV-equipped arrivals in the terminal area from all simulation runs are shown in Figure 4. The RNP-equipped arrivals are indicated in light green. Figure 4a shows results of the baseline conditions next to those using the TSS system, Figure 4b. Most of the scheduled delay in the Center was no more than two minutes. Schedule conformance at the meter fix was within 10 seconds at the $75^{\text {th }}$ percentile and 30 seconds at the $99^{\text {th }}$ under all conditions. achievable when Center controllers use the TMA-TM schedule to pre-condition traffic properly, regardless of whether the TSS TRACON advisories were displayed. Peak and average throughput increased by two to four aircraft per hour when the TSS system was used. Deviation from the PBN routes was an average of 2.73 and $1.31 \mathrm{NM}$ less per aircraft when using TSS in Scenarios B-1 and B-2 respectively. There was an average of 1.79 and $0.88 \mathrm{NM}$ less flight distance flown per aircraft in Scenarios B-1 and B-2 when using the TSS system. The total number of

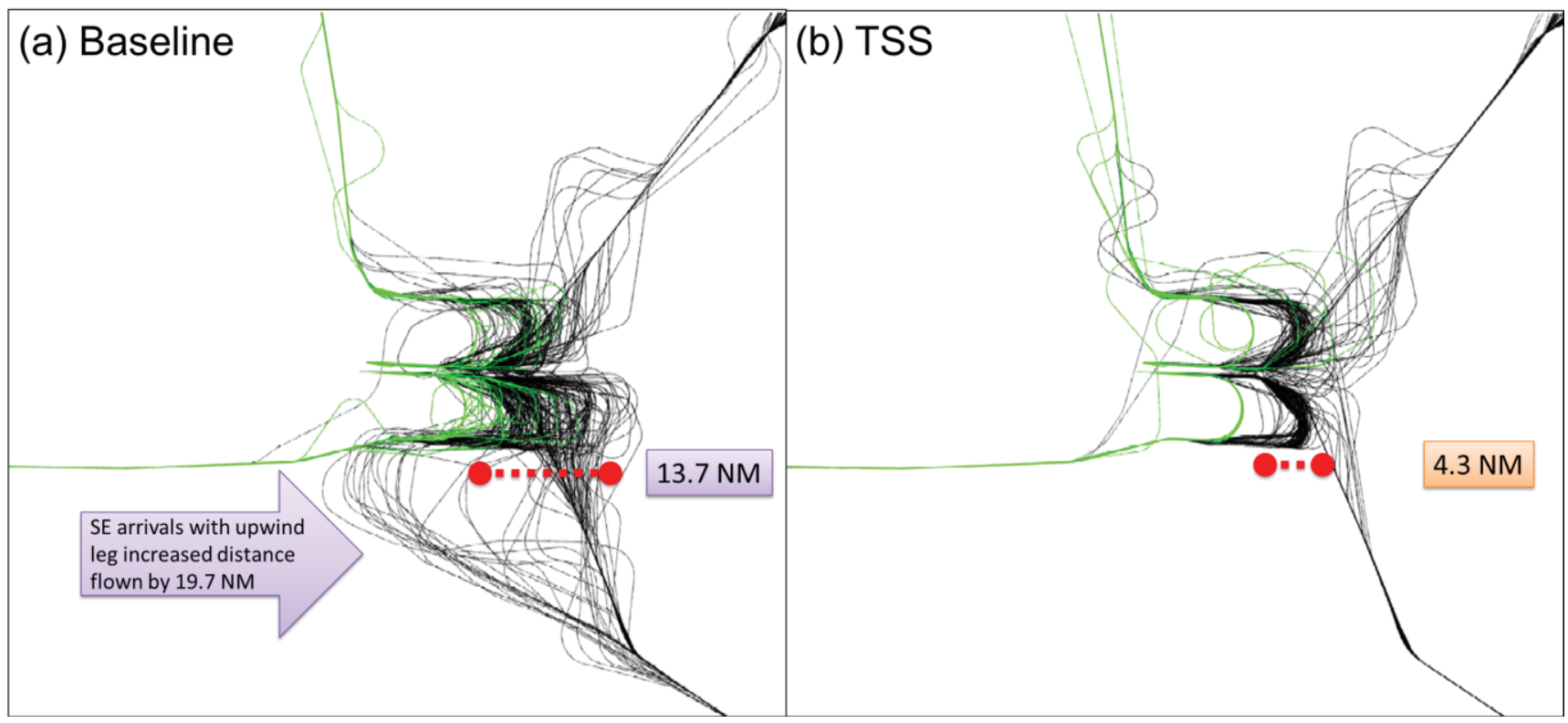

Figure 4. Lateral paths of RNAV/RNP-equipped jets in the (a) baseline case and (b) when using TSS.

TRACON controllers delay or expedite an aircraft intercepting the final approach by extending or compressing the downwind or base leg. Using the TSS system, the base extension spread was $4.3 \mathrm{NM}$ compared to $13.7 \mathrm{NM}$ in the baseline case. There is also an apparent upwind leg built in the South Feeder, which was not needed in the conditions having the TSS system. Southeast arrivals with the upwind leg flew an average of $19.7 \mathrm{NM}$ extra path distance when the TSS system was not used.

The Visual Flight Rule (VFR) arrival rate for PHX in the West Flow configuration is 78 aircraft per hour defined by the FAA $7210.4 \mathrm{~B}$. The average throughput ranged from 77-86 and peak values were 86-96 aircraft per hour under all test conditions, regardless of whether the TSS system was used. Throughput $\mathrm{w}$ as estimated using a 10-minute sliding window. This suggests that the PHX VFR rate is aircraft that had level segments longer than 60 seconds in the terminal area was found to be about $5 \%$ less with the TSS system. The average time spent in level flight was reduced by 39 seconds when using TSS, from 187 to 148 seconds.

Figure 5 shows the percentage of RNP-equipped aircraft remaining on the RNP-AR approach without controller intervention. Data are shown when using the TSS system versus the baseline case for Scenarios B-1 and B-2. When the TSS system was used, RNPequipped aircraft were taken off its RNP-AR approach less often than the baseline case. Nearly all RNP-equipped aircraft remained on the RNP-AR approach. Aircraft were taken off the RNP-AR approach if there were perceived separation issues. These aircraft flew similar path distances as the rest of the RNAV-equipped aircraft. 


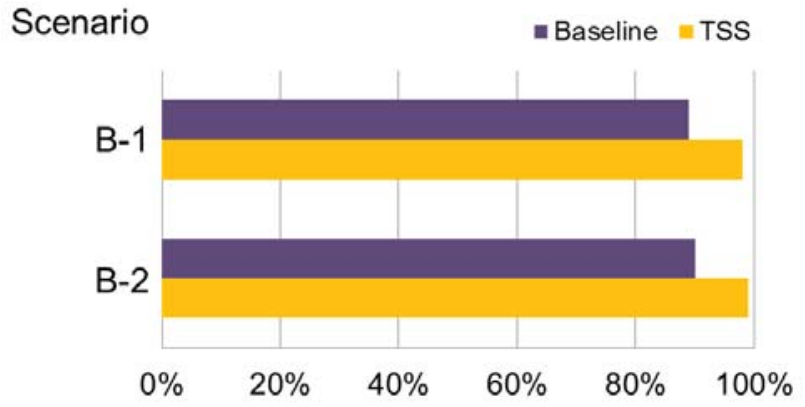

Figure 5. Percentage of uninterrupted RNP-AR approaches for Scenario $B$.

The number of controller clearances issued was estimated based on recorded pilot entries. Figure 6 plots the different clearance types categorized by equipage level. Data from Scenarios B-1 and B-2 were combined. The number of clearances was reduced 9\% overall for both RNP and RNAVequipped aircraft. Final controllers issued an average of 1.3 fewer clearances per aircraft when using the TSS system. In the baseline case, more heading and altitude clearances were issued by Finals to merge flows and also account for interrupted RNP-AR approaches. Feeder controllers had an overall increase of 0.4 clearances per aircraft. More speed clearances were issued by Feeder Controllers when the TSS system was used, since the tools often advised reducing aircraft speed earlier than usual to condition the flow to final. The number of controller clearances issued to RNP-equipped versus RNAVequipped aircraft was reduced by at least $40 \%$ in the Final sectors.

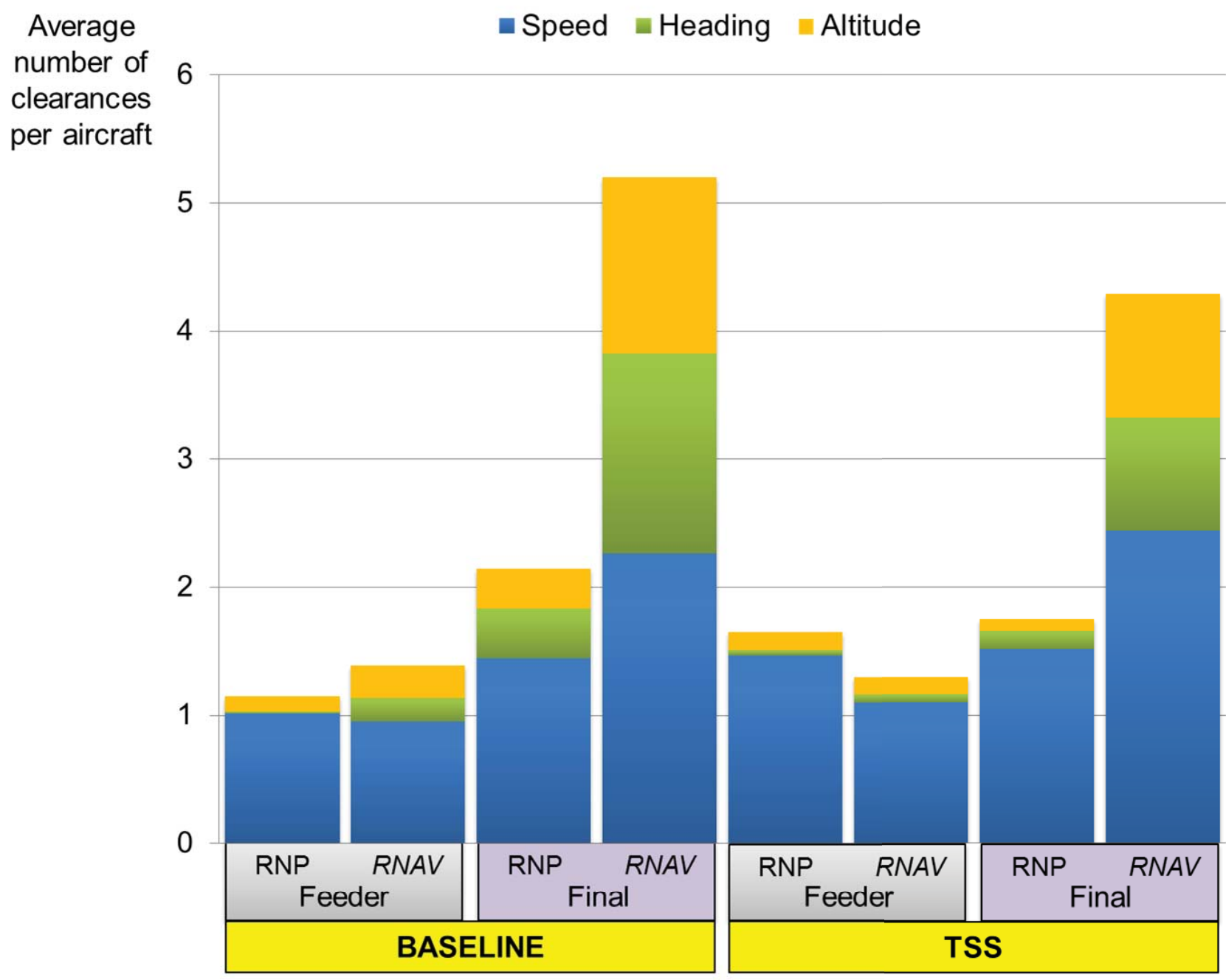

Figure 6. Number of controller clearances issued per aircraft. 


\section{Learning Effects}

Scenario A was experienced by both groups of controllers with different levels of TSS experience, ref. Table 2. Data were collected from the 'New' controller participants after they had completed 14 simulation runs using Scenario B; during 7 of these runs they used the TSS system. The 'Experienced' controller participants had at least 4 weeks of previous experience with the TSS system and data were collected using Scenario A only. Results between the 'Experienced' and 'New' controller participants were evaluated to examine effects of tool familiarity.

Figure 7 displays the lateral paths of the RNAVequipped arrivals in the terminal area using Scenario A. The RNP-equipped arrivals are indicated in light green. Results shown are from using the TSS system versus the baseline condition for both groups of controller participants. The difference in controller technique in merging the RNP and RNAV arrivals is apparent in the South sectors, where the upwind leg is not used by the 'Experienced' participants. In the

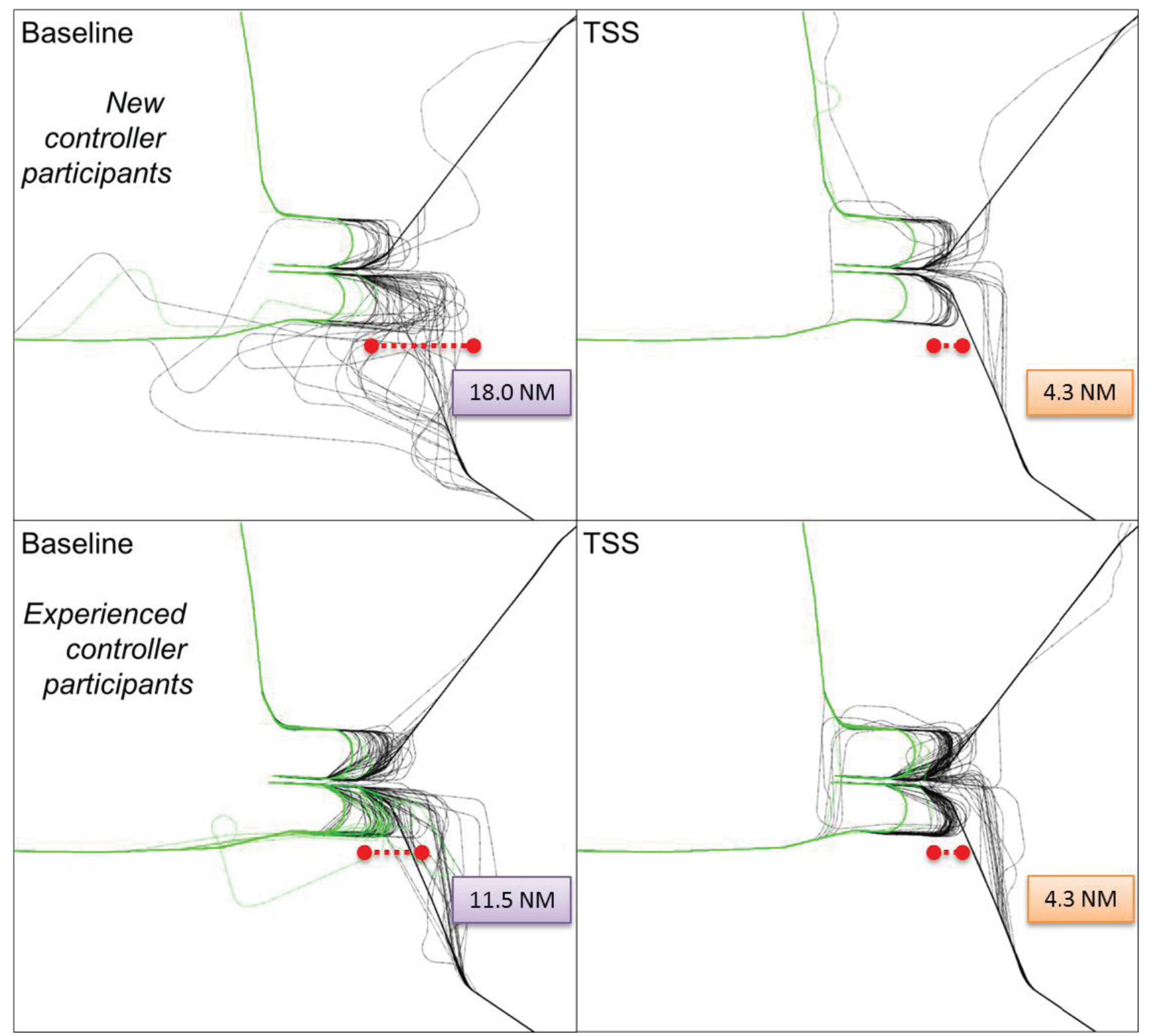

Figure 7. Lateral paths of RNAV/RNP-equipped jets for both sets of controller participants. 
baseline conditions, the base extension range was 11.5 NM for the 'Experienced' participants and 18.0 NM for 'New' participants. When the TSS system was used, the spread was $4.4 \mathrm{NM}$ for both sets of controllers.

The average throughput rate for both sets of participants increased by three aircraft per hour when using the TSS system. With the TSS system, 'New' participants had an average/peak throughput of $82 / 93$ aircraft per hour versus 85/96 by 'Experienced' participants. In conditions with the TSS system in use, the average aircraft path distance was about the same for both groups of participants.

Figure 8 shows the percentage of uninterrupted RNP-AR approaches when using the TSS system versus the baseline condition for both sets of controllers. The number of uninterrupted RNP-AR approaches was greater when the TSS system was used. The 'New' participants were able to consistently achieve the same percentage levels for Scenario A and B (ref. Fig. 5). How ever, for Sc enario A, without the TSS system, the average aircraft path distance increased by $4.79 \mathrm{NM}$ for the 'New' participants. The high level of RNP adherence was at the expense of increased path distance flown by the non-RNP equipped aircraft.

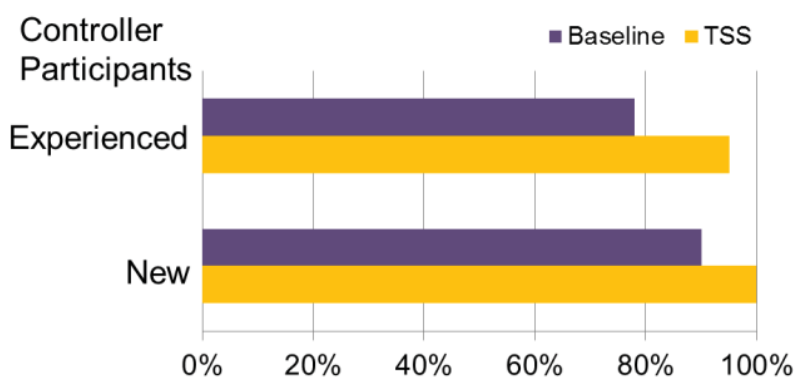

\section{Figure 8. Percentage of uninterrupted RNP-AR by two sets of controller participants.}

The 'Experienced' controllers had higher percentages of interrupted RNP-AR approaches overall, despite having higher throughput rates. This may be due to differences in training. In previous HITL simulations with the 'Experienced' controllers, maintaining high throughput rates was emphasized as opposed to Best-Equipped, Best-Served (BEBS), which was the training focus for the 'New' participants. In conditions where the 'Experienced' participants used the TSS system, the average aircraft path distance actually increased by $0.99 \mathrm{NM}$ over the baseline cases. Without the TSS tools, they shortened the downwind leg of the RNAV arrivals to maintain throughput, especially for the less loaded runway 26 (ref. Table 1). The TSS system balanced the runways and also positioned RNAV arrivals to merge more efficiently with the RNP-equipped arrivals conducting RNP-AR approaches. The PBN route structure definition used in the TSS system, however, has a slightly longer downwind leg when compared to the one used by the 'Experienced' participants in the baseline case. The participants followed the TSS advisories based on the longer route, resulting in the increase in distance flown compared to the baseline condition.

The TSS system advisories change the default runway for a subset of aircraft, and are designed to improve the overall efficiency of operations. Runway changes were adhered to $99 \%$ of the time by both sets of participants. Scenario A typically had four to five changes per run and Scenario B usually had one. In the baseline conditions, both sets of controller participants rarely initiated runway changes (i.e., at most one) in either scenario. When they did, the average extra path distance of the crossover compared to remaining on the default runway was 18.3 NM for the baseline conditions. Following the TSS system runway advisories, the average total extra path distance per crossover was reduced to 6.1 NM.

The penalty of extra path distance for balancing the runways is partially offset by the benefit of increasing the number of uninterrupted RNP-AR approaches. Figure 9 shows the total number of RNPAR approaches that could not be completed when using the TSS system and the baseline condition. This data includes both sets of participants using Scenario A and is categorized by runway. In the baseline case, there were 13 RNP-AR approaches that could not be completed. Of those, $85 \%$ were on the more heavily loaded South runway $25 \mathrm{~L}$. The coordination required for merging crossovers, however, should also be considered. When the TSS system was used, $75 \%$ could not complete the RNPAR approach on the North runway 26. The larger number of interrupted RNP-AR approaches on 26 may be due to the additional complexity in merging the over-the-airport crossovers from $25 \mathrm{~L}$. 


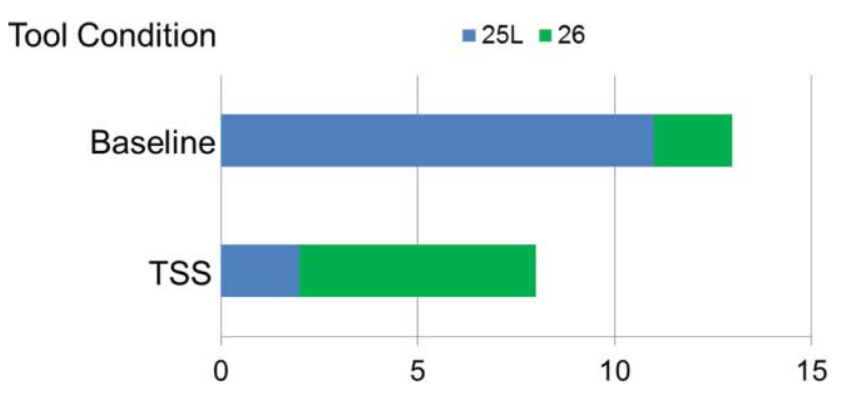

Figure 9. Number of interrupted RNP-AR approaches per runway for Scenario $A$.

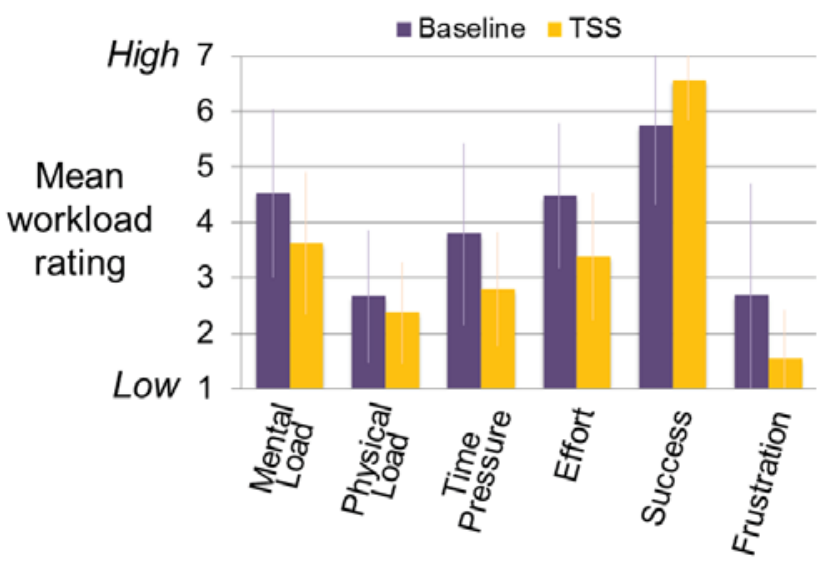

Figure 10. Mean workload rating for NASA TLX subscales for 'New' controllers.

For the 'Experienced' participants, there were higher mean workload ratings reported in the baseline conditions, but no differences were statistically significant. The 'Experienced' participants provided written comments on what type of events increased their workload. These events included not having the TSS tools available and taking an aircraft off its RNP-AR approach, which resulted in additional vectoring to merge with the rest of the traffic. There were also notes on two aircraft performing over-theairport crossovers simultaneously, which required additional verbal coordination for separation.

'New' controllers similarly rated the manageability of the operations on a 7 point scale. The traffic mix was rated as "easy to manage" ( mean $=6.5$, st. $\underline{\text { dev. }}=0.9$ ) when using the TSS tools, but only "reasonable to manage" $(\mathrm{m}=4.9, \mathrm{sd}=1.7)$ without them. Controller responses indicated that the traffic mix was easier to manage when having the TSS system, supporting the indications of lower workload in Figure 10. The difference in means was more than 1.5 scale-points, which is statistically signific ant at the $\mathrm{p}<0.001$ level.

The delivery of traffic from the Center controllers was "excellent, very easy to work" $(\mathrm{m}=6.8, \mathrm{sd}=0.6)$ under all conditions. When the TSS schedule was used, the STAs were reported to be "easy to achieve" $\quad(\mathrm{m}=6.4, \quad \mathrm{sd}=0.9)$. The 'Experienced' participants also reported less verbal coordination was initiated from TRACON and Center. In the baseline conditions, the need for verbal coordination was reported in $75 \%$ of the controller 
responses. Of the $75 \%$, more than 10 aircraft had to be coordinated at least a third of the time. When the TSS system was used, the need for verbal coordination was reported in only $50 \%$ of the controller responses. When it was necessary, fewer than 10 aircraft needed coordination.

The usage frequency of the TSS tools is presented in Figure 11. The most popular tool was the slot marker, with participants reporting using them in all simulation runs. Most tools were new to the controllers prior to the HITL simulations, but were used more than $80 \%$ of the time. The most frequently used tools are complimentary with each other. Controllers attempted to pair up the slot marker and aircraft target by comparing the two IAS and determining the appropriate speed adjustment. Speed advisories were rarely used directly; they were understood to represent a reasonable estimate given the forecasted winds and anticipated slot marker trajectory that controllers would tailor in order to account for other factors. The slot marker positioning was consistent with the sequence number and runway assignment. Controllers used the sequence number to facilitate planning of the merges, especially of the RNP and RNAV-equipped aircraft near final. The runway assignment by TMA-TM was adhered to $99 \%$ of the time.

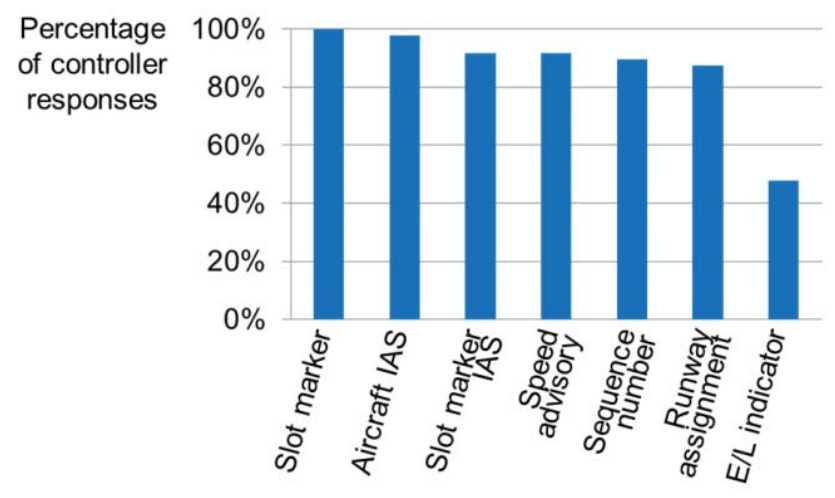

Figure 11. TSS tool usage for 'New' controllers.

There were several current-day STARS tools that were available for controller use during all test conditions. When the TSS tools were available, the STARS tools were used the least. In the baseline conditions, the current-day STARS tools were generally used more, but still reported as being used in less than half of the controller responses overall. The 'New' and 'Experienced' participants had similar tool usage ratings.
'New' controllers also reported on the number of path stretch maneuvers and results are shown in Figure 12. Figure 12 illustrates that there was less vectoring when the TSS system was used compared to the baseline condition. RNP-equipped aircraft were not vectored nearly $90 \%$ of the time when the TSS system was used versus $70 \%$ in the baseline conditions. Those that were not RNP-equipped had a similar reduction in path stretch maneuvers from $60 \%$ to $25 \%$. Results also show that aircraft with RNP equipage were issued $30-35 \%$ fewer path stretch maneuvers compared to aircraft without advanced equipage. Among all aircraft that were vectored, the most frequent amount of vectoring increased from one to two to four times in the baseline condition. Results were consistent with those shown in Figure 6.

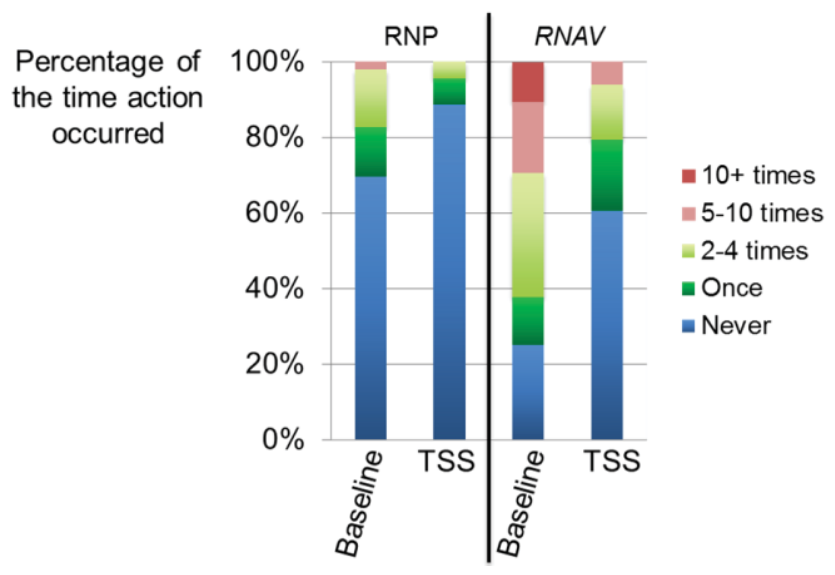

Figure 12. Frequency of path stretch maneuvers for 'New' controllers.

'New' controllers reported on the most common reasons for issuing path stretch maneuvers. The most frequently reported reason was to maintain safe separation, $57 \%$ of the time when using the TSS system and $69 \%$ in the baseline condition. In the baseline condition, the second most common reason was to give preference to an RNP-equipped aircraft, reported $41 \%$ of time. When the TSS system was used, the need for vectoring to give an RNP-equipped aircraft preference dropped to $10 \%$.

\section{Conclusion}

NASA has developed the TSS system, a suite of advanced arrival management technologies combining time-based scheduling and controller precision spacing tools. TSS is a ground-based controller automation tool that facilitates sequencing 
and merging arrivals on terminal PBN routes. TSS aims to maintain PBN operations, especially at highly congested airports. The benefits of using PBN are in predictable flight paths that can be designed to improve capacity and efficiency in operations, while supporting environmental initiatives such as reducing noise, emissions, and fuel consumption.

In collaboration with the FAA and MITRE, the TSS system performance was evaluated with a typical terminal routing infrastructure under saturated traffic demand levels. Traffic scenarios had mixed RNAV/RNP equipage, where the more advanced RNP-equipped aircraft had preferential treatment with a shorter approach option. Forty high-fidelity HITL simulation runs were conducted at NASA Ames Research Center's ATC lab with currently active CPC participants who had little to no experience using TSS prior to the simulations. Results were also compared with controller participants who had more experience with TSS, which provided insight on the effects of additional experience.

Simulation results indicate the TSS system demonstrated benefits in enabling PBN, while maintaining high throughput rates-10\% above baseline demand levels. When the TSS system was used, flight path predictability improved. The severity of path deviation was reduced by two NM and the variance in the downwind leg length reduced by $75 \%$. Arrivals flew more fuel-efficient descents for longer, spending 39 seconds less in level altitude segments. TSS was able to more efficiently merge arrivals with mixed RNAV/RNP equipage. RNPequipped arrivals were able to better capitalize on the benefits of advanced equipage, where less vectoring was needed and 99\% of RNP-AR approaches were uninterrupted. The TSS system also reduced controller workload ratings, with statistically significant differences at the $\mathrm{p}<0.01$ level for most of the TLX subscales.

Controller participants with less TSS experience succeeded in maintaining an equivalent level of uninterrupted RNP-AR approaches without TSS, but aircraft ended up with an average of four NM extra path distance. Participants with more experience with the TSS system achieved slightly higher throughput, but had more interrupted RNP-AR approaches. Both groups of controller participants reported similar TSS tool preferences. These results indicate that the benefits that can be achieved with the TSS system are influenced by training and controller technique more so than TSS experience. Nevertheless, controller participants who had the least amount of TSS training and usage were still able to realize notable benefits when using the system.

NASA transferred the TSS technical requirements to the FAA in the Fall of 2013. The TSS system is a promising candidate for near-term operational use to enable PBN, since the capabilities were embedded in TMA, which is operational at Centers nationwide. The TSS advisory tools have also been prototyped in the operational STARS system. MITRE has completed an independent assessment of TSS and published a comprehensive analysis report of the HITL simulation results [23]. NASA and MITRE will continue further research to finalize TSS requirements for field evaluation and deployment.

\section{References}

[1] Federal Aviation Administration (FAA), 2010, Fact Sheet - NextGen Goal: Performance-Based Navigation.

[2] The MITRE Corporation, 2009, NextGen: Area Navigation (RNAV) and Required Navigation (RNP), Statement of Dr. Agam N. Sinha, Committee on Transportation and Infrastructure, Subcommittee on Aviation, U.S. House of Representatives.

[3] FAA, 2013, NextGen Implementation Plan.

[4] Becher, T., Barker, D., Smith, A., 2004, Methods for Maintaining Benefits for Merging Aircraft on Terminal RNAV Routes, $23^{\text {rd }}$ Digital Avionics Systems Conference (DASC), Salt Lake City, UT.

[5] Mundra, A., Bateman, H., Smith A., et. al., 2011, Converging Runway Display Aid in the NAS: Challenges, Successes and Outlook, 30 ${ }^{\text {th }}$ DASC, Seattle, WA.

[6] Smith, A., Becher, T., 2005, A Study of SPACR Ghost Dynamics Applied to RNAV Routes in the Terminal Area, $24^{\text {th }}$ DASC, Washington, D.C.

[7] Mac Williams, P., Porter, D., An Assessment of a Controller Aid for Merging and Sequencing Traffic on Performance-Based Arrival Routes, unknown. 
[8] MacWilliams, P., A Site Study and Benefit Analysis of a TRACON Situational Awareness Aid, 2007, $7^{\text {th }}$ AIAA Aviation Technology, Integration and Operations Conference (ATIO), Belfast, Northern Ireland.

[9] Shepley, J. P., 2008, Analysis of Potential Delay Reduction from Implementation of the Relative Position Indicator (RPI) at Operation Evolution Partnership (OEP) Airports, MP080060, The MITRE Corporation, McLean, VA.

[10] Hopper, J., 2011, Analysis of a Wind Compensation Tool for the Relative Position Indicator (RPI), $30^{\text {th }}$ DASC, Seattle, WA.

[11] Atkins, S., Capozzi, B., 2011, Relative Position Indicator for Merging Mixed RNAV and Vectored Arrival Traffic, $30^{\text {th }}$ DASC, Seattle, WA.

[12] Swenson, H., Thipphavong, J., Martin, L., et. al., 2011, Design and evaluation of the Terminal Area Precision Scheduling and Spacing System, 9th USA/Europe ATM R\&D Seminar, Berlin, Germany.

[13] Kupfer, M., Callantine, T., Martin, L., et. al., 2011, Controller support tools for schedule-based terminal-area operations, 9th USA/Europe ATM R\&D Seminar, Berlin, Germany.

[14] Callantine, T., Palmer, E., Kupfer, M., et. al., 2010, Human-in-the-loop Simulation of Trajectorybased Terminal-area Operations, 27th International Congress of the Aeronautical Sciences (ICAS), Nice, France.

[15] Martin, L., Swenson, H., Sadovsky, A., et. al., 2011, Effects of Scheduling and Spacing tools on Controller's Performance and Perceptions of their Workload, 30th DASC, Seattle, WA.

[16] Mercer, J., Callantine, T., Martin, L., 2012, Resolving Off-Nominal Situations in Schedule-Based Terminal Area Operations: Results from a HumanIn-The-Loop Simulation, 28 ${ }^{\text {th }}$ ICAS, Brisbane, Australia.

[17] Swenson, H., Jung, J., Martin, L., et. al., 2012, Development and Evaluation of the Terminal Precision Scheduling and Spacing System for Offnominal Condition Operations, 31 st DASC, Williamsburg, VA.
[18] Jung, J., Swenson, H. N., Martin, L., et. al, 2013, Evaluation of the Terminal Area Precision Scheduling and Spacing System for PerformanceBased Navigation Arrivals, $32^{\text {nd }}$ DASC, Syracuse, NY.

[19] Prevot, T., 2012, How to Compute A Slot Marker - Calculation of Controller-Managed Spacing Tools for Efficient Descents with Precision Scheduling, $31^{\text {st }}$ DASC, Williamsburg, VA.

[20] Prevot, T., MACS: A Simulation Platform for Today's and Tomorrow's Air Traffic Operations, 2007, AIAA Modeling and Simulation Technologies Conference and Exhibit, Hilton Head, SC.

[21] Wynnyk, C., 2013, Meteorological Accuracy for Aviation Applications, Contract No. DTFAWA-10-C00080, McLean, VA, The MITRE Corporation.

[22] Hart, S. G., Staveland, L. E., 1988, Development of the NASA-TLX (Task Load Index): Results of Empirical and Theoretical Research, Amsterdam, P. Hancock \& N. Meshkati (Eds.), Human mental workload, pp. 139-183.

[23] Wynnyk, M., Kopald, H., 2013, Terminal Sequencing and Spacing (TSS) Simulation 2 Post Analysis Report, Project No. 0212HE01-1F, McLean, VA, The MITRE Corporation.

\section{Acknowledgments}

The authors would like to thank the FAA and NATCA controller participants, Danny Vincent and the Human Solutions Inc. team in developing the controller training materials and assistance during the HITL simulations. The authors also appreciate the invaluable expertise of Neil Hightower, John Nolan, Tom Rudolph, Mark Spaulding, and Tom Wood for training controller participants and providing feedback on airspace design and procedures.

\section{2nd Digital Avionics Systems Conference October 6-10, 2013}

